\title{
Study of Anatomical Variations of Mitral Papillary Muscles and their Significance: A Cadaveric Study from Southern India
}

\section{ABSTRACT}

Introduction: Papillary Muscles ( $\mathrm{PaM})$ are the muscular components of the mitral apparatus and include a small portion of the wall of the Left Ventricle (LV). There are Anterolateral Papillary Muscles (ALPM) and Posteromedial Papillary Muscles (PMPM) that are usually single, though may be seen in groups or as accessory PaM. The mitral papillary muscle is usually conical in shape with a single base and single apex, though they may also be pyramidal, fan-shaped or broad-apexed which may influence the passage of blood flow. Various patterns like bifid-apexed, perforated or long PaM may also be present with variable length. Alterations in the left ventricular shape and size may lead to disturbances in the Mitral Valve (MV) function. A papillary muscle, like the MV complex is unique and differs in morphology from one individual with high variability. Due to above mentioned reasons, knowledge of variations of mitral papillary muscle is crucial.

Aim: To study variations in the morphology of mitral PaM in terms of length, number, shape and pattern in adult human cadaveric hearts.

Materials and Methods: A total of fifty adult heart specimens, without gross deformity, were taken from the Institute of Anatomy,
Madras Medical College and studied by conventional dissection method. The presence of mitral PaM, their number, length, shape and pattern were studied. A data sheet was made using Microsoft excel software and the mean, percentages and mode of variables were calculated.

Results: In the present study, it was found that the two mitral $\mathrm{PaM}$, namely anterolateral and PMPM were present in all the specimens. PMPM had a mean length of $21.6 \pm 4 \mathrm{~mm}$ and were longer than ALPM that had a mean of $18.9 \pm 3.97 \mathrm{~mm}$. PMPM (68\%) were mostly present in groups and ranged between one and four whereas the ALPM was predominantly single (76\%). Most AMPM (56\%) and PMPM (60\%) were cone shaped. A $40 \%$ of ALPM and $60 \%$ PMPM had single, undivided base with an undivided apex.

Conclusion: Considerable variations in number, shape, pattern and length of the PaM observed in the present study correlated with the findings of most other studies done previously. The data obtained may be useful for radiologists and cardio-thoracic surgeons.

\section{INTRODUCTION}

The heart has four chambers and the left atrium receives oxygenated blood from the lungs which contracts to fill the LV through the left AV orifice guarded by the MV. The MV is comprised of the annulus, the anterior and posterior leaflets, two PaM, Chordae Tendinae (CT) which connect the leaflets with the PaM. PaM are the muscular components of the mitral apparatus and include a small portion of the wall of the LV [1]. The PaM are two in number occupying the anterolateral and posteromedial portions of the LV, though they may be present in multiple groups [2]. Accessory PaM other than ALPM and PMPM may be present [3]. The papillary muscle is conical in shape with undivided base and an undivided apex [1]. Other shapes like pyramidal, fanshaped, broad apexed and cylindrical have been observed and patterns like split base, perforated or long PaM may also be present with variable length $[1,4,5]$. The shape of mitral PaM largely influences the passage of the blood flow [4]. Alterations in the left ventricular shape and size may alter the position of the papillary muscle leading to disturbances in the MV function [6]. Sometimes the apex may be divided into two or three presenting as bifid or trifid apices.

Papillary Muscles (PaM), like the MV complex is unique and differs in morphology from one individual to another with a high variability $[7,8]$. Longer mitral PaM may be associated with hypertrophic obstructive cardiomyopathies [9]. Large, anomalous papillary muscle originating from the posterior wall may cause mid-ventricular obstruction. In such a case, smaller papillary muscle elements originate from the central muscle core and course radially to the walls of the LV like arms. Such a papillary muscle is called the octopus papillary muscle
[10]. Due to above mentioned reasons, valvular diseases are more common on the left side than on the right. Therefore, knowledge of variations of mitral papillary muscle is crucial for surgical procedures involving MV [11].

Thus, the study aimed to evaluate the variations in the morphology of mitral PaM in terms of length, number, shape and pattern in adult human cadaveric hearts.

\section{MATERIALS AND METHODS}

This descriptive study included 50 formalin-fixed hearts of adult human cadavers that were taken from the Institute of Anatomy, Madras Medical College. The study was done during August 2017October 2017, after getting clearance from the Institutional Ethics Committee of Madras Medical College (no-27072016).

Inclusion criteria: Apparently normal-looking heart specimens without gross deformities irrespective of age and sex were included for the study.

Exclusion criteria: Abnormally enlarged and grossly deformed hearts were excluded.

The samples were analysed by conventional dissection method. The LV was opened by an incision extending from the left auricle down to the apex along the left border in order to view the PaM. Blood clots were washed away. The presence, number of groups, length, shape, and pattern of the left ventricular PaM were observed. The length of the PaM was measured using digital vernier calipers. The length was measured from the origin of the papillary muscle at the base to the point of attachment to the CT. In the present study, 
cone-shaped PaM with single base and a single apex arising from middle one-third of the left ventricular wall were considered to be normal. Those with separate bases were considered to be groups of PaM and those with divided apex were considered as bifid or trifid PaM based on the numbers [Table/Fig-1-7].

\section{STATISTICAL ANALYSIS}

All the recorded values were entered and a data sheet was made using Microsoft excel software.The mean, mode and percentages were calculated for all variables i.e., length, number, shape and pattern of papillary muscles of the mitral valve complex.

\section{RESULTS}

Length of papillary muscles of the mitral valve complex: Length of the left ALPM ranged from 6.30 to $27.55 \mathrm{~mm}$ with a mean of $18.9 \pm 3.97 \mathrm{~mm}$ [Table/Fig-1]. The length of left PMPM ranged from 9.19 to $28.98 \mathrm{~mm}$ with a mean of $21.6 \pm 4 \mathrm{~mm}$.

Number of papillary muscles of the mitral valve complex: The two PaM namely the anterolateral and posteromedial muscles were present in all the specimens. The ALPM was mostly found single, whereas the PMPM was mostly present in groups [Table/Fig-8].

Shape of papillary muscles of the mitral valve complex: In the present study, the ALPM and PMPM of the LV were mostly conical [Table/Fig-4] in shape, second common being pyramidal [Table/Fig-5]. Others were cylindrical and fan-shaped [Table/Fig-9].

Pattern of papillary muscles of the mitral valve complex: Among AMPM and PMPM groups, single muscle having undivided apex with an undivided base (classical type) was mostly seen. Second common pattern was single base with bifid apex [Table/Fig-7].
Other patterns like divided base with fused apex, perforated [Table/ Fig-6], small projections were also seen [Table/Fig-10].

\section{DISCUSSION}

The MV has always been a common topic for studies due to its functional importance. Very few studies have been done on the morphology of mitral PaM. The number of PaM is highly variable according to Victor and Nayak, who studied the variations in mitral $\mathrm{PaM}$ and stated that the MV apparatus, including the PaM, is as unique to each individual as one's own fingerprints [13].

Length of papillary muscles: Shree B et al., stated that the mean length of ALPM decreased as the number of groups increased [11]. Among PMPM groups, the mean length of bellies decreased as number of heads increased. This was consistent with the study done by Aulakh KK et al., where the mean length of PMPM was 2.3 $\mathrm{cm}$ and was found to be larger in males than in females [8]. The mean length of PMPM was also found to decrease as the number of heads increased. Mohammadi $S$ et al., reported that ALPM (1.9 $\mathrm{cm})$ was longer than PMPM $(1.1 \mathrm{~cm})$ [12]. In the present study, the mean length of left PMPM $(2.16 \mathrm{~cm})$ was more than that of the ALPM $(1.9 \mathrm{~cm})$.

The variations in papillary muscle may influence the pathophysiological effects of various disorders. During surgery the chordae can be studied by traction on the concerned papillary muscle. Similarly, very long chordae in a homograft may be prone to rupture and very short chordae may favour early fibrous fusion between the papillary muscle and the leaflet tissue [13]. Longer mitral PaM may be associated with hypertrophic obstructive cardiomyopathies [9].

Number of groups: In the present study, the ALPM was predominantly single (76\%) whereas the PMPM were seen as two
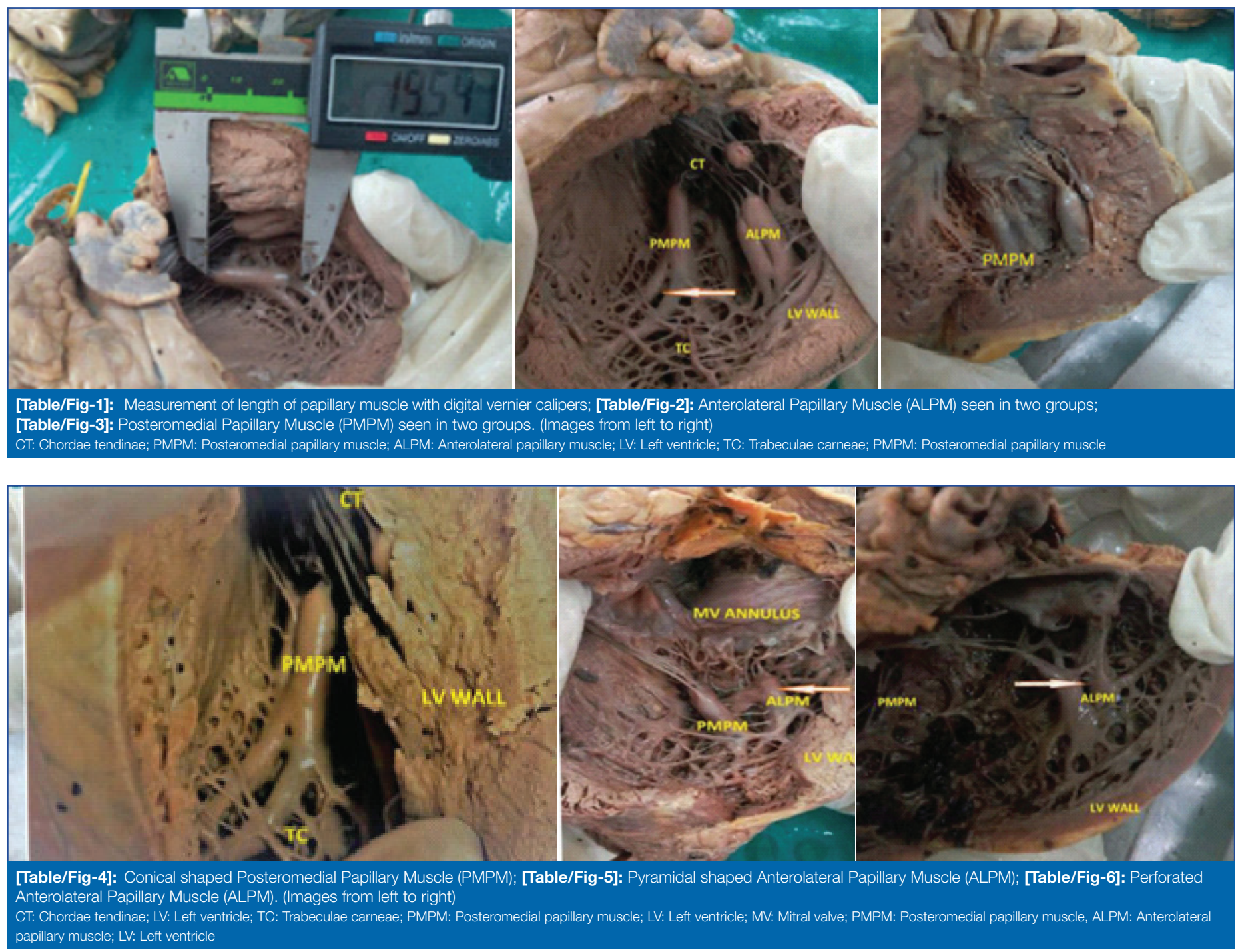




[Table/Fig-7]: Anterolateral Papillary Muscle (ALPM) showing single base with bifid apex.

\begin{tabular}{|l|c|c|c|c|}
\hline $\begin{array}{l}\text { Papillary } \\
\text { muscle }\end{array}$ & $\begin{array}{c}\text { Single } \\
\text { N (\%) }\end{array}$ & $\begin{array}{c}\text { Two groups } \\
\text { N (\%) }\end{array}$ & $\begin{array}{c}\text { Three groups } \\
\text { N (\%) }\end{array}$ & $\begin{array}{c}\text { Four groups } \\
\text { N (\%) }\end{array}$ \\
\hline Anterolateral & $38(76 \%)$ & $12(24 \%)$ & Nil & Nil \\
\hline Posteromedial & $16(32 \%)$ & $28(56 \%)$ & $5(10 \%)$ & $1(2 \%)$ \\
\hline
\end{tabular}

[Table/Fig-8]: Number of groups of Papillary Muscles (PaM) of the Mitral Valve (MV) complex.

\begin{tabular}{|l|c|c|}
\hline $\begin{array}{l}\text { Shape of papillary } \\
\text { muscle }\end{array}$ & $\begin{array}{c}\text { Anterolateral papillary } \\
\text { muscles N (\%) }\end{array}$ & $\begin{array}{c}\text { Posteromedial papillary } \\
\text { muscle N (\%) }\end{array}$ \\
\hline Conical & $28(56 \%)$ & $30(60 \%)$ \\
\hline Pyramidal & $16(32 \%)$ & $8(16 \%)$ \\
\hline Cylindrical & $4(8 \%)$ & $5(10 \%)$ \\
\hline Fan-shaped & $2(4 \%)$ & Nil \\
\hline Broad-apexed & Nil & $7(14 \%)$ \\
\hline TTable/Fig-9]: Shapes of papillary muscles of the mitral valve complex.
\end{tabular}

\begin{tabular}{|l|c|c|}
\hline $\begin{array}{l}\text { Pattern of papillary } \\
\text { muscle }\end{array}$ & $\begin{array}{c}\text { Anterolateral papillary } \\
\text { muscles N (\%) }\end{array}$ & $\begin{array}{c}\text { Posteromedial papillary } \\
\text { muscle N (\%) }\end{array}$ \\
\hline Classical & $20(40 \%)$ & $30(60 \%)$ \\
\hline Single base, bifid apex & $14(28 \%)$ & $8(16 \%)$ \\
\hline Divided base, fused apex & $8(16 \%)$ & $6(12 \%)$ \\
\hline Perforated & $5(10 \%)$ & $2(4 \%)$ \\
\hline $\begin{array}{l}\text { Base attached to a large } \\
\text { bridge }\end{array}$ & Nil & Nil \\
\hline Small projections & $3(6 \%)$ & . \\
\hline [Table/Fig-10]: Patterns of the papillary muscles of the mitral valve complex.
\end{tabular}

groups (56\%); three groups (10\%) and four groups in $2 \%$. Rusted IE et al., conducted a study on MV commissures and reported that ALPM was single in most specimens whereas, PMPM was mostly in groups of two or more [14]. Similarly, Shree B et al., also reported single ALPM in $86 \%$ whereas single PMPM were seen only in $14 \%$ and rest of PMPM were in groups of two or more [11]. However, Georghitescu $\mathrm{G}$ et al., stated that both anterolateral and PMPM were mostly seen in groups of two (60\%) [2].

Gunnal SA et al., described four categories of mitral PaM- single ALPM and single PMPM were found only in 3\% specimens [15]; others were seen in two or more groups. In their study, both anterolateral and PMPM were mostly seen in multiple groups.
An increased number of left ventricular PaM may cause outflow obstruction [15]. Single papillary muscle is usually associated with other malformations like supramitral valve ring, diffuse subaortic stenosis, coarctation of aorta [11]. An accessory papillary muscle is usually of functional significance because of its association with congenital mitral regurgitation [16].

Shape of papillary muscles: In the present study, ALPM of the LV were mostly conical (56\%), second common shape being pyramidal (32\%). The PMPM were also mostly conical (60\%). Pyramidal was the second most common form seen among the PaM.

Bose $\mathrm{P}$ et al., in their study reported that PaM mostly, broad-apexed (52\%) which was consistent with study findings of Gunnal SA et al., where both anterolateral and PMPM groups were mostly broad apexed (37\%) and the classical cone shape was seen as second common (34\%) [15, 17]. This was contradictory to study done by Georghitescu $\mathrm{G}$ et al., in which papillary muscle was mostly conical (63\%), cylindrical being second common (19\%) and broad-apexed in very few specimens (9\%) [2].

The shape of the left ventricular PaM largely influences the flow of blood [5]. The chances of left ventricular outflow tract obstruction are more in hypertrophy of PaM, especially associated with fanshaped muscles and broad apexed PaM [4]. On the other hand, conical shape facilitates blood flow, posing minimum obstruction to the outflow tract. The PaM that best facilitates cardiovascular physiology by posing minimum obstruction to blood flow is conical shaped, broad based, attached to the ventricular wall away from the centre of cavity [15].

Pattern of papillary muscles: In the present study, among anterolateral muscle groups, classical type with single base and single apex was observed in 40\% specimens while, 60\% PMPM had a classical pattern. Single base with bifid apex was observed as second common variant (28\% ALPM and 16\% PMPM). Classical type was found to be more common among both ALPM and PMPM in the present study. This was consistent with the study done by Gunnal SA et al., in which it was stated that classical pattern, where there is a single, undivided apex with an undivided base most prevalent (36\%) Second common pattern was separate bases with fused apex (21\%) [15]. According to Bose P et al., separate bases with fused apex was most commonly observed (37\%) and classical type only as second common variant (31\%) [17]. Other variants like single base with divided apex, small projections of PaM, Perforated PaM were also described in their study.

Large, anomalous papillary muscle originating from the posterior wall may cause mid-ventricular obstruction. In such a case, smaller papillary muscle elements originate from the central muscle core and course radially to the walls of the LV like arms or tentacles in a transverse plane, dividing the left ventricular cavity into an apical and a sub aortic chamber. Such a papillary muscle is called the Octopus Papillary Muscle [10].

Anomalous insertion of one or both left ventricular PaM directly into the anterior mitral leaflet without interposition of the CT may be largely responsible for outflow tract obstruction seen in hypertrophic obstructive cardiomyopathy [18]. Recognition of this anomalous insertion leading to outflow obstruction in such patients may have important implications with regard to planning operative strategy.

\section{Limitation(s)}

The present study was done on formalin-fixed hearts from cadavers of unknown sex. Hence, sex differences could not be studied. Further, the findings were based on observation only. Imaging studies of PaM on living subjects may have more validity.

\section{CONCLUSION(S)}

According to the present study, both ALPM and PMPM were always present and were mostly conical with classical pattern. PMPM were 
mostly seen in groups and were longer than ALPM. The variations may have ethnic relevance and may give more information if further studies are done. To conclude, no two PaM had similar structure, each one seemed to be unique.

\section{Acknowledgement}

We would like to thank and pay respect to all those kind hearts who have donated their body for research and education purposes without whom this work would not have been accomplished. I wish to express exquisite thankfulness and gratitude to my most respected teacher, Dr. Sudha Seshayyan, Director and Professor, Institute of Anatomy, Madras Medical College for her valuable guidance and persistent support.

\section{REFERENCES}

[1] Susan Standring. Gray's Anatomy. The Anatomical Basis of Clinical Practice, $40^{\text {th }}$ edition, 2008.

[2] Gheorghitescu R, Toba M, lliescu DM, Bordei P. Anatomical consideration of the number and form of the papillary muscle in the left ventricle. ARS Medica Tomitana. 2016;22(2):119-27.

[3] Chaudhary S. A rare morphological variant accessory posterolateral cusp of mitral valve with accessory papillary muscle in left ventricle in an adult cadaver in middle east-a case report. J Am Sci. 2017;13(3).

[4] Ozan H, Kocabıyılk N, Demirel B, Yalçın B, Cömert A. Pattern of connection between papillary muscle and chordae tendineae of left ventricle. Gulhane Med J. 2012;54(4):275-78

[5] Lakhanpal AV, Shrivastava SK, Verma SK. Study of types of papillary muscles of mitral valve in central Indians. SSN Online 2393-915X ICV 5043. 2016;3(9):2454-7379.
[6] Kono T, Sabbah HN, Rosman H, Alam M, Jafri S, Goldstein S. Left ventricular shape is the primary determinant of functional mitral regurgitation in heart failure. J Am Coll Cardiol. 1992;20(7):1594-98.

[7] Krawczyk-Ożóg, Hołda MK, Bolechała F, Sorysz D, Dudek D. Anatomy of mitra subvalvular apparatus. pdf [Internet]. JTCVS.2018;155(5):2002-10

[8] Aulakh KK, Saxena AK, Aneja PS, Bansal S. A morphometric study of the papillary muscles of the left ventricles of human cadaveric hearts in North-West Indian population. J Evol Med Dent Sci. 2016;5(100):7346-53.

[9] Madu EC, Baugh DS, D Cruz IA, Johns C. Left ventricular papillary muscle morphology and function in left ventricular hypertrophy and left ventricular dysfunction. Med Sci Monit. 2001;7(6):1212-18.

[10] Shah AS, Kukar A, Chaudhry FA, Sherrid MV. Unusual anomalous single papillary muscle causing symptomatic mid-left ventricular cavity obstruction: Octopus papillary muscle. J Am Soc Echocardiogr. 2006;19(7):939-e9.

[11] Shree B, Singla RK, Sharma RK, Kumar A. A study of papillary muscles of the left ventricle in the adult human cadavers. Int J Anat Res. 2016:4(2):2285-93.

[12] Mohammadi S, Hedjazi A, Sajjadian M, Ghoroubi N, Mohammadi M, Erfani S. Study of the normal heart size in Northwest part of Iranian population: A cadaveric study. J Cardiovasc Thorac Res. 2016;8(3):119.

[13] Victor S, Nayak VM. Variations in the papillary muscles of the normal mitral valve and their surgical relevance. Journal of cardiac surgery. 1995;10(5):597-607.

[14] Rusted IE, Scheifly CH, Edwards JE. Studies of the mitral valve. I. Anatomic features of the normal mitral valve and associated structures. Circulation. 1952;6(6):825-31.

[15] Gunnal SA, Farooqui MS, Wabale RN. Study of mitral valve in human cadaveric hearts. Heart Views Off J Gulf Heart Assoc. 2012;13(4):132.

[16] Rim Y, McPherson DD, Kim H. Effect of congenital anomalies of the papillary muscles on mitral valve function. J Med Biol Eng. 2015;35(1):104-12.

[17] Bose P, Singh R. Morphological variations of papillary muscles in North Indians: A cadaveric study. J Anat Sciences. 2015; 23(2):01-05.

[18] Klues HG, Roberts WC, Maron BJ. Anomalous insertion of papillary muscle directly into anterior mitral leaflet in hypertrophic cardiomyopathy. Significance in producing left ventricular outflow obstruction. Circulation. 1991;84(3):1188-97.

\section{PARTICULARS OF CONTRIBUTORS:}

1. Assistant Professor, Department of Anatomy, Government Tirunelveli Medical College, Nagercoil, Tamil Nadu, India.

2. Assistant Professor, Department of Anatomy, K.A.P. Viswanatham Government Medical College, Trichy, Tamil Nadu, India.

NAME, ADDRESS, E-MAIL ID OF THE CORRESPONDING AUTHOR:

S Valli,

No. 215/18, Arunachalam Colony, Asambu Road, Vadasery,

Nagercoil, Tamil Nadu, India.

E-mail: svalli145@gmail.com

\section{AUTHOR DECLARATION:}

- Financial or Other Competing Interests: None

- Was Ethics Committee Approval obtained for this study? Yes

- Was informed consent obtained from the subjects involved in the study? NA

- For any images presented appropriate consent has been obtained from the subjects. NA
PLAGIARISM CHECKING METHODS: Jain $\mathrm{H}$ et al.]

- Plagiarism X-checker: Apr 02, 2021

- Manual Googling: May 24, 2021

- iThenticate Software: Jun 25, 2021 (9\%)
ETYMOLOGY: Author Origin

Date of Submission: Mar 31, 2021 Date of Peer Review: May 03, 2021

Date of Acceptance: May 25, 2021 Date of Publishing: Oct 01, 2021 